SIR: The article by Lawrence et al (Journal, September 1991, 159, 334-340) has prompted us to share some of our results concerning hospital admissions in the psychiatry of mental handicap.

Our uncontrolled retrospective survey has shown that with the changes in admission policies in the mental handicap hospitals, the rate and number of people coming to those hospitals has dropped. At the Cell Barnes Hospital, the number of new admissions for $1979(n=156)$ and $1980(n=198)$ were quite different from the number of new admissions for $1989(n=64)$ and $1990(n=33)$, showing a significant fall to between one-third and one-fifth in the past decade.

There have been several factors influencing the fall in the number of admissions in the large mental handicap hospitals. An awareness of reasons for hospital admissions and its consequences, the benefits versus drawbacks of admissions, cessations of easy availability of beds, and the decrease of social and indiscriminate admissions have affected the number of people coming to hospital.

An awareness of benefits of community care, the increases in community resources, rehabilitation, respite care, day-time facilities, and adult training centres, and the increase in the number of other professionals have eased the follow-up of people with dual diagnosis and multiple problems who had been candidates for admissions in the past.

In the psychiatry of mental handicap, we now believe that community alternatives to hospital admissions have crystallised the needs for the real admissions of people with psychiatric and behavioural disorders. We are also aware that although alternatives to in-patient care have a valid place in the psychiatry of mental handicap, those alternatives cannot fully replace the assessment, treatment aspects, and security of the hospital.

We now have a better understanding and clearer definition of the mentally handicapped people who can live in a highly supported environment in the community as compared with the few whose intractable challenging behaviour and dual diagnosis make them candidates to become new long-stay hospital patients.

The issue now is to increase the community facilities at district level provided by health and social services to accommodate all the patients who can live in the community and to resettle all the hospital residents who can cope in the community environment.

Our results have shown that in mental handicap, community care has reduced the need for psychiatric beds and has brought more insight into new admissions; these results will be sustained if the community provides the necessary resources for people to live independently.

Contrary to the information coming from adult services, when it comes to admissions of mentally handicapped people, the bed-rock of illness will reach stability and clarification after community care has been fully implemented. At today's pace this may take many years.

Charing Cross and Westminster Medical School

D. KOHEN St Dunstan's Road

London W6 8RP

S. Attale

Cell Barnes Hospital

G. MATHEW

St Albans

\section{Family psychiatric histories in general practice} referrals

SIR: The role of heredity in many psychiatric disorders is now acknowledged. With the advent of new biological techniques, molecular genetics will offer a range of approaches to psychiatric science (Baron \& Rainer, 1988). Hence there is renewed interest in the genetics of psychiatric illness. General practitioners have traditionally been viewed as family doctors, and they are in a unique position to report the occurrence of psychiatric illnesses within the family. Family psychiatric history has been identified as one of the key items to be included in a referral letter from a general practitioner to a psychiatrist (Pullen \& Yellowlees, 1985).

In this context, we report one finding from an audit of referrals to a small psychiatric team in an inner city area of Nottingham. During a six-month period beginning in October 1990, 41 referrals were received from general practitioners. Forty of these referrals failed to mention the presence or absence of a family psychiatric history.

There could be many reasons for such a high rate of omission of the details of family psychiatric history. Perhaps general practitioners did not consider family histories to be relevant. Perhaps they assumed that psychiatrists were already aware of seriously affected family members. Many of the referral letters received were computerised, and we wondered whether the fixed format of these led to the family history not being included.

We would welcome comments from psychiatrists on their experiences of practice elsewhere, and also as to how they perceive this finding.

BARON, M. \& RAINER, J. D. (1988) Molecular genetics and human disease, implication for modern psychiatric research and practice. British Journal of Psychiatry, 152, 741-747. 